DOI 10.1007/s11141-015-9565-6

Radiophysics and Quantum Electronics, Vol.57, No.11, April, 2015

(Russian Original Vol. 57, No. 11, November, 2014)

\title{
ELECTRODYNAMIC CHARACTERISTICS OF A STRIP ANTENNA LOCATED ON A PLANE INTERFACE OF A RESONANT MAGNETOPLASMA AND AN ISOTROPIC MEDIUM
}

\author{
T. M. Zaboronkova, ${ }^{1,2 *}$ A. S. Zaitseva, ${ }^{1}$ A. V. Kudrin, ${ }^{1}$ \\ and B. Spagnolo ${ }^{1,3,4}$
}

UDC 533.951

\begin{abstract}
We study the electrodynamic characteristics of an antenna having the form of an infinitesimally thin, perfectly conducting narrow strip located on a plane interface of a resonant magnetoplasma and an isotropic medium. The antenna is perpendicular to an external magnetic field and is excited by a given voltage. Singular integral equations for the antenna current, on the basis of which the current distribution is found in the case of an infinitely long radiator, are obtained. The limits of applicability of an approximate method based on the transmission line theory for determining the current distribution and input impedance of the antenna are established. Within the framework of this method, the results obtained are generalized to the case of a finite-length strip antenna.
\end{abstract}

\section{INTRODUCTION}

Electrodynamic characteristics of metal antennas in a magnetoplasma have been studied in a large number of publications (see, e.g., [1-4] and references therein). Interest in this problem is due, in particular, to the wide use of antenna systems in different experiments conducted in the space and laboratory plasmas. Most of the theoretical papers on the subject consider the antennas located in a homogeneous magnetoplasma (see, e. g., [5-8]). Recently, increased attention has been paid to the study of antenna characteristics in inhomogeneous plasma media $[4,9-11]$. The case where the antenna is located near the interface of two media (in particular, isotropic and magnetized) is of the greatest interest since the presence of such an interface can significantly affect the current distribution and power characteristics of the sources.

In this paper, using the integral equation method, we solve a model problem of the current distribution and input impedance of a strip antenna that is perpendicular to an external magnetic field and located on a plane interface of an isotropic medium and a magnetoplasma. The main attention is paid to the case of a resonant plasma medium where the refractive index of one of the normal modes tends to inifinity for a certain angle between the wave vector and the direction of the external magnetic field $[1,4]$. Such conditions take place in many experimental situations.

\section{FORMULATION OF THE PROBLEM AND BASIC RELATIONS}

Consider an infinitely long antenna, which is oriented along the $x$ axis of a Cartesian coordinate system and has the form of a perfectly conducting, infinitesimally thin, narrow strip of width $2 d$ lying in the

* t.zaboronkova@rambler.ru

${ }^{1}$ N. I. Lobachevsky State University of Nizhny Novgorod; ${ }^{2}$ R. E. Alekseev State Technical University of Nizhny Novgorod, Nizhny Novgorod, Russia; ${ }^{3}$ Università di Palermo and CNISM, Unità di Palermo, Palermo;

${ }^{4}$ Istituto Nazionale di Fisica Nucleare, Sezione di Catania, Catania, Italy. Translated from Izvestiya Vysshikh Uchebnykh Zavedenii, Radiofizika, Vol.57, No. 11, pp. 889-901, November 2014. Original article submitted July 14, 2014; accepted November 13, 2014.

0033-8443/15/5711-0795 (C) 2015 Springer Science+Business Media New York 


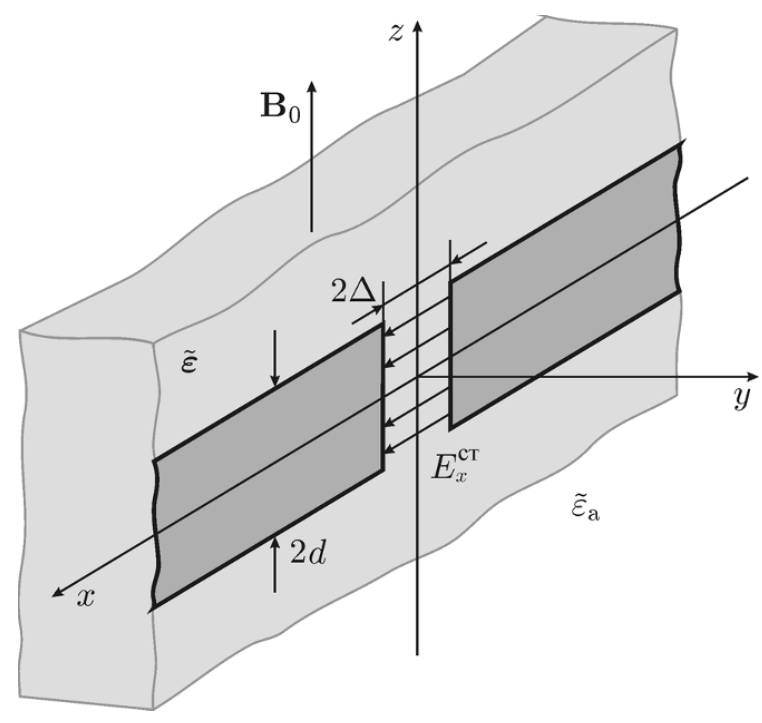

Fig. 1. Geometry of the problem.

$x z$ plane (see Fig. 1). It is assumed that this plane coincides with the interface of a magnetoplasma and an isotropic medium. The external magnetic field $\mathbf{B}_{0}$ is aligned with the $z$ axis. The half-space $y<0$ is filled with a homogeneous cold collisionless magnetoplasma, whose dielectric permittivity tensor has the form

$$
\tilde{\varepsilon}=\epsilon_{0}\left(\begin{array}{ccc}
\varepsilon & -i g & 0 \\
i g & \varepsilon & 0 \\
0 & 0 & \eta
\end{array}\right)
$$

where $\epsilon_{0}$ is the electric constant. Expressions for the elements of tensor (1) are given in, e. g., [12]. Recall that the plasma is resonant in the frequency ranges in which the relationship $\operatorname{sign} \varepsilon \neq \operatorname{sign} \eta$ is fulfilled for the diagonal elements of the dielectric permittivity tensor $[1,4]$. In particular, the whistler frequency range

$$
\omega_{\mathrm{LH}}<\omega<\omega_{H}<\omega_{\mathrm{p}},
$$

where $\omega_{\mathrm{LH}}$ is the lower hybrid frequency, and $\omega_{H}$ and $\omega_{\mathrm{p}}$ are the gyrofrequency and plasma frequency of electrons, respectively, which is considered in what follows, is resonant. Note that this frequency range is also important in many problems related to the use of antennas in plasmas [1, 4]. Homogeneous medium in the half-space $y>0$ is isotropic and has a dielectric permittivity $\tilde{\varepsilon}_{\mathrm{a}}=\epsilon_{0} \varepsilon_{\mathrm{a}}$. In the case where the medium in the region $y>0$ is free space, one should put $\varepsilon_{\mathrm{a}}=1$.

The current in the antenna is excited by a time-harmonic (proportional to the factor $\exp (i \omega t)$ ) given voltage that creates an electric field with the only component $E_{x}^{\text {ext }}$, which is nonzero for $y=0$ and $|z|<d$ in a narrow interval ("gap") $|x| \leq \Delta$ :

$$
E_{x}^{\mathrm{ext}}(x, 0, z)=\frac{V_{0}}{2 \Delta}[U(x+\Delta)-U(x-\Delta)][U(z+d)-U(z-d)] .
$$

Here, $V_{0}=$ const is the complex amplitude of the voltage applied to the gap, $\Delta$ is the gap half-width, and $U$ is a Heaviside function. The distribution of $E_{x}^{\text {ext }}$ for $|z|<d$ can be represented as the following expansion into a Fourier integral:

$$
E_{x}^{\operatorname{ext}}(x, 0, z)=\frac{k_{0}}{2 \pi} \int_{-\infty}^{+\infty} \mathcal{E}_{x}^{\operatorname{ext}}\left(n_{x}\right) \exp \left(-i k_{0} n_{x} x\right) \mathrm{d} n_{x}
$$

where

$$
\mathcal{E}_{x}^{\mathrm{ext}}\left(n_{x}\right)=V_{0} \frac{\sin \left(k_{0} n_{x} \Delta\right)}{k_{0} n_{x} \Delta},
$$

$k_{0}=\omega / \mathrm{s}$ is the wave number in free space, and $c$ is the light speed.

The density $\mathbf{J}$ of the electric current excited in the antenna by an external field given by Eq. (3) will be sought in the form

$$
\mathbf{J}=\mathbf{x}_{0} I(x, z) \delta(y)
$$

where $|z|<d$, and $\delta(y)$ is a Dirac function. The surface density $I(x, z)$ of the current obviously admits the following representation:

$$
I(x, z)=\frac{k_{0}}{2 \pi} \int_{-\infty}^{+\infty} \mathcal{I}\left(n_{x}, z\right) \exp \left(-i k_{0} n_{x} x\right) \mathrm{d} n_{x} .
$$


To find the distribution $I(x, z)$, we express the tangential components $E_{x}$ and $E_{z}$ of the electric field excited by current (6) via the Fourier transform $\mathcal{I}\left(n_{x}, z\right)$ of the surface current density and take into account boundary conditions for the field components on the interface $y=0$. In addition, we make use of boundary conditions for the tangential components of the electric field on the antenna surface $(y=0$ and $|z|<d)$ :

$$
\begin{gathered}
E_{x}+E_{x}^{\mathrm{ext}}=0, \\
E_{z}=0 .
\end{gathered}
$$

This procedure provides integral equations for the unknown quantity $\mathcal{I}\left(n_{x}, z\right)$ and thus reduces the problem of determining the antenna current to the solution of the corresponding integral equations.

\section{DERIVING INTEGRAL EQUATIONS FOR THE ANTENNA CURRENT}

At first we obtain expressions for the components of the field corresponding to current density (7) from the system of Maxwell equations. For the region $y<0$ the Maxwell equations can be written in the form

$$
\operatorname{rot} \mathbf{E}=-i \omega \mu_{0} \mathbf{H}, \quad \operatorname{rot} \mathbf{H}=i \omega \tilde{\varepsilon} \mathbf{E},
$$

where $\mu_{0}$ is the magnetic constant. Replacing the dielectric permitivity tensor $\tilde{\varepsilon}$ by the quantity $\tilde{\varepsilon}_{\mathrm{a}}$, we obtain the Maxwell equation for the region $y>0$.

The solution of the equations for the electromagneic field can be written in the form

$$
\begin{aligned}
& \mathbf{E}(x, y, z)=\frac{k_{0}}{2 \pi} \int_{-\infty}^{+\infty} \mathbf{E}\left(n_{x}, y, z\right) \exp \left(-i k_{0} n_{x} x\right) \mathrm{d} n_{x}, \\
& \mathbf{H}(x, y, z)=\frac{k_{0}}{2 \pi} \int_{-\infty}^{+\infty} \mathbf{H}\left(n_{x}, y, z\right) \exp \left(-i k_{0} n_{x} x\right) \mathrm{d} n_{x},
\end{aligned}
$$

where the quantities $\mathbf{E}\left(n_{x}, y, z\right)$ and $\mathbf{H}\left(n_{x}, y, z\right)$ admit, in turn, the representation

$$
\begin{aligned}
\mathbf{E}\left(n_{x}, y, z\right) & =\frac{k_{0}}{2 \pi} \int_{-\infty}^{+\infty} \mathbf{E}\left(n_{x}, y, n_{z}\right) \exp \left(-i k_{0} n_{z} z\right) \mathrm{d} n_{z}, \\
\mathbf{H}\left(n_{x}, y, z\right) & =\frac{k_{0}}{2 \pi} \int_{-\infty}^{+\infty} \mathbf{H}\left(n_{x}, y, n_{z}\right) \exp \left(-i k_{0} n_{z} z\right) \mathrm{d} n_{z} .
\end{aligned}
$$

Note that Eqs. (11) and (12) give expansions of the field into Fourier integrals over the wave numbers $n_{x}$ and $n_{z}$ normalized to $k_{0}$. Then the quantities $E_{x}\left(n_{x}, y, n_{z}\right), E_{y}\left(n_{x}, y, n_{z}\right), H_{x}\left(n_{x}, y, n_{z}\right)$, and $H_{y}\left(n_{x}, y, n_{z}\right)$ can conveniently be expressed via the components $E_{z}\left(n_{x}, y, n_{z}\right)$ and $H_{z}\left(n_{x}, y, n_{z}\right)$, which satisfy the following system of equations for $y<0$ :

$$
\begin{gathered}
\hat{L} E_{z}-k_{0}^{2} \frac{\eta}{\varepsilon}\left(n_{z}^{2}-\varepsilon\right) E_{z}=-i k_{0}^{2} \frac{g}{\varepsilon} n_{z} Z_{0} H_{z}, \\
\hat{L} H_{z}-k_{0}^{2}\left(n_{z}^{2}+\frac{g^{2}-\varepsilon^{2}}{\varepsilon}\right) H_{z}=i k_{0}^{2} \frac{g}{\varepsilon} \eta n_{z} Z_{0}^{-1} E_{z},
\end{gathered}
$$

where

$$
\hat{L}=\frac{\partial^{2}}{\partial y^{2}}-\left(k_{0} n_{x}\right)^{2}
$$


and $Z_{0}=\left(\mu_{0} / \epsilon_{0}\right)^{1 / 2}$ is the wave impedance of free space. For the region $y>0$, one should put $\varepsilon=\eta=\varepsilon_{\mathrm{a}}$ and $g=0$ in Eqs. (13) and (14). Solutions for the fields should satisfy the radiation conditions at infinity (for $|y| \rightarrow \infty$ ), as well as the boundary conditions for the tangential field components on the interface $y=0$. For the corresponding Fourier-transformed field components, we have

$$
\begin{gathered}
E_{x}\left(n_{x}, y-0, n_{z}\right)=E_{x}\left(n_{x}, y+0, n_{z}\right), \quad E_{z}\left(n_{x}, y-0, n_{z}\right)=E_{z}\left(n_{x}, y+0, n_{z}\right), \\
H_{x}\left(n_{x}, y-0, n_{z}\right)=H_{x}\left(n_{x}, y+0, n_{z}\right), \quad H_{z}\left(n_{x}, y-0, n_{z}\right)=H_{z}\left(n_{x}, y+0, n_{z}\right)-\mathcal{I}\left(n_{x}, n_{z}\right),
\end{gathered}
$$

where we introduced the rotation

$$
\mathcal{I}\left(n_{x}, n_{z}\right)=\int_{-d}^{d} \mathcal{I}\left(n_{x}, z^{\prime}\right) \exp \left(i k_{0} n_{z} z^{\prime}\right) \mathrm{d} z^{\prime} .
$$

It is seen from Eq. (16) that the boundary conditions consist in the continuity of the tangential field components $E_{x}, E_{z}$, and $H_{x}$ on the interface $y=0$. As concerns $H_{z}$, this component is continuous on the interface for $|z|>d$, while for $|z|<d$ it should undergo a jump corresponding to the surface current (6). Hence, the jump of the Fourier transform $H_{z}\left(n_{x}, y, n_{z}\right)$ for $y=0$ in Eq. (16) is determined by the quantity $\mathcal{I}\left(n_{x}, n_{z}\right)$ calculated from Eq. (17).

It can easily be found that the Fourier transform $\mathbf{E}\left(n_{x}, y, n_{z}\right)$ and $\mathbf{H}\left(n_{x}, y, n_{z}\right)$ of the electromagnetic field components can be written as

$$
\begin{gathered}
E_{x}\left(n_{x}, y, n_{z}\right)=i \sum_{k=1}^{2} B_{k} \frac{i n_{y, k}+\alpha_{k} n_{x}}{n_{\perp k}} \exp \left(i k_{0} n_{y, k} y\right), \\
E_{y}\left(n_{x}, y, n_{z}\right)=-\sum_{k=1}^{2} B_{k} \frac{i \alpha_{k} n_{y, k}+n_{x}}{n_{\perp k}} \exp \left(i k_{0} n_{y, k} y\right), \\
E_{z}\left(n_{x}, y, n_{z}\right)=\frac{i}{\eta} \sum_{k=1}^{2} B_{k} n_{\perp k} n_{k} \exp \left(i k_{0} n_{y, k} y\right), \\
H_{x}\left(n_{x}, y, n_{z}\right)=-Z_{0}^{-1} \sum_{k=1}^{2} B_{k} n_{k} \frac{i n_{y, k}-\beta_{k} n_{x}}{n_{\perp k}} \exp \left(i k_{0} n_{y, k} y\right), \\
H_{y}\left(n_{x}, y, n_{z}\right)=i Z_{0}^{-1} \sum_{k=1}^{2} B_{k} n_{k} \frac{i \beta_{k} n_{y, k}-n_{x}}{n_{\perp k}} \exp \left(i k_{0} n_{y, k} y\right), \\
H_{z}\left(n_{x}, y, n_{z}\right)=-Z_{0}^{-1} \sum_{k=1}^{2} B_{k} n_{\perp k} \exp \left(i k_{0} n_{y, k} y\right)
\end{gathered}
$$

for $y<0$, and as

$$
\begin{gathered}
E_{x}\left(n_{x}, y, n_{z}\right)=-\frac{C n_{y}+D n_{x} n_{z}}{n_{\perp}^{2}} \exp \left(-i k_{0} n_{y} y\right), \\
E_{y}\left(n_{x}, y, n_{z}\right)=\frac{C n_{x}-D n_{y} n_{z}}{n_{\perp}^{2}} \exp \left(-i k_{0} n_{y} y\right), \\
E_{z}\left(n_{x}, y, n_{z}\right)=D \exp \left(-i k_{0} n_{y} y\right), \\
H_{x}\left(n_{x}, y, n_{z}\right)=\frac{-C n_{x} n_{z}+\varepsilon_{\mathrm{a}} D n_{y}}{Z_{0} n_{\perp}^{2}} \exp \left(-i k_{0} n_{y} y\right), \\
H_{y}\left(n_{x}, y, n_{z}\right)=-\frac{C n_{y} n_{z}+\varepsilon_{\mathrm{a}} D n_{x}}{Z_{0} n_{\perp}^{2}} \exp \left(-i k_{0} n_{y} y\right),
\end{gathered}
$$




$$
H_{z}\left(n_{x}, y, n_{z}\right)=Z_{0}^{-1} C \exp \left(-i k_{0} n_{y} y\right)
$$

for $y>0$. Here, $B_{k}, C$, and $D$ are the coefficients to be determined $(k=1,2)$. The other quantities in Eqs. (18) and (19) are described by the formulas

$$
\begin{aligned}
& n_{\perp k}^{2}=(2 \varepsilon)^{-1}\left\{\varepsilon^{2}-g^{2}+\varepsilon \eta-(\eta+\varepsilon) n_{z}^{2}+(-1)^{k}\left\{(\eta-\varepsilon)^{2} n_{z}^{4}\right.\right. \\
& \left.\left.+2\left[g^{2}(\eta+\varepsilon)-\varepsilon(\eta-\varepsilon)^{2}\right] n_{z}^{2}+\left(\varepsilon^{2}-g^{2}-\varepsilon \eta\right)^{2}\right\}^{1 / 2}\right\} \\
& n_{\perp}^{2}=\varepsilon_{\mathrm{a}}-n_{z}^{2}, \quad n_{k}=-\frac{\varepsilon}{n_{z} g}\left[n_{z}^{2}+n_{\perp k}^{2}\left(n_{z}\right)+\frac{g^{2}}{\varepsilon}-\varepsilon\right], \quad \alpha_{k}=\left[n_{z}^{2}+n_{\perp k}^{2}\left(n_{z}\right)-\varepsilon\right] / g, \\
& \beta_{k}=n_{z} / n_{k}, \quad n_{y}=\left(\varepsilon_{\mathrm{a}}-n_{x}^{2}-n_{z}^{2}\right)^{1 / 2}, \quad n_{y, k}=\left[n_{\perp k}^{2}\left(n_{z}\right)-n_{x}^{2}\right]^{1 / 2} .
\end{aligned}
$$

In order to satisfy the radiation condition at infinity (for $|y| \rightarrow \infty$ ), the branches of the functions $n_{y}$ and $n_{y, k}$ in Eq. (20) should be chosen as follows:

$$
\operatorname{Im} n_{y}<0, \quad \operatorname{Im} n_{y, k}<0 .
$$

The coefficients $B_{k}, C$, and $D$ in Eqs. (18) and (19) can be determined from boundary conditions (16):

$$
\begin{gathered}
B_{1}=\frac{Z_{0} \mathcal{I}\left(n_{x}, n_{z}\right)}{\Delta\left(n_{x}, n_{z}\right) n_{\perp 1}}\left[i h_{2} \frac{\eta}{\varepsilon_{\mathrm{a}}} \frac{n_{2} n_{y}}{n_{\perp}^{2}}-e_{2} \frac{\eta}{\varepsilon_{\mathrm{a}}} \frac{n_{x} n_{z}}{n_{\perp}^{2}}-n_{2} \frac{\varepsilon_{\mathrm{a}}-n_{x}^{2}}{\varepsilon_{\mathrm{a}} n_{\perp}^{2}}\right], \\
B_{2}=\frac{Z_{0} \mathcal{I}\left(n_{x}, n_{z}\right)}{\Delta\left(n_{x}, n_{z}\right) n_{\perp 2}}\left[-i h_{1} \frac{\eta}{\varepsilon_{\mathrm{a}}} \frac{n_{1} n_{y}}{n_{\perp}^{2}}+e_{1} \frac{\eta}{\varepsilon_{\mathrm{a}}} \frac{n_{x} n_{z}}{n_{\perp}^{2}}+n_{1} \frac{\varepsilon_{\mathrm{a}}-n_{x}^{2}}{\varepsilon_{\mathrm{a}} n_{\perp}^{2}}\right], \\
C=\frac{Z_{0} \mathcal{I}\left(n_{x}, n_{z}\right)}{\Delta\left(n_{x}, n_{z}\right)}\left[\frac{\eta}{\varepsilon_{\mathrm{a}}}\left(h_{1}-h_{2}\right) \frac{n_{x} n_{z}}{n_{\perp}^{2}}+\frac{i n_{y}}{n_{\perp}^{2}}\left(n_{2} e_{1}-n_{1} e_{2}\right)+\frac{\eta}{\varepsilon_{\mathrm{a}}}\left(n_{2} e_{1} h_{2}-n_{1} e_{2} h_{1}\right)\right], \\
D=\frac{Z_{0} \mathcal{I}\left(n_{x}, n_{z}\right)}{\Delta\left(n_{x}, n_{z}\right)}\left[-\frac{\eta}{\varepsilon_{\mathrm{a}}}\left(h_{1}-h_{2}\right) \frac{n_{y}}{n_{\perp}^{2}}+\frac{i}{\varepsilon_{\mathrm{a}}}\left(n_{2} e_{1}-n_{1} e_{2}\right) \frac{n_{x} n_{z}}{n_{\perp}^{2}}\right],
\end{gathered}
$$

where

$$
\begin{aligned}
\Delta\left(n_{x}, n_{z}\right)=n_{2}\left[\frac{\eta}{\varepsilon_{\mathrm{a}}} e_{1} h_{2}+\frac{i n_{y}}{n_{\perp}^{2}}\left(e_{1}+\frac{\eta}{\varepsilon_{\mathrm{a}}} h_{2}\right)\right]-n_{1}\left[\frac{\eta}{\varepsilon_{\mathrm{a}}} e_{2} h_{1}+\frac{i n_{y}}{n_{\perp}^{2}}\left(e_{2}+\frac{\eta}{\varepsilon_{\mathrm{a}}} h_{1}\right)\right] \\
-\left(n_{2}-n_{1}\right) \frac{\varepsilon_{\mathrm{a}}-n_{x}^{2}}{\varepsilon_{\mathrm{a}} n_{\perp}^{2}}+\frac{\eta}{\varepsilon_{\mathrm{a}}} \frac{n_{x} n_{z}}{n_{\perp}^{2}}\left(e_{1}+h_{1}-e_{2}-h_{2}\right) ; \\
e_{k}=\frac{i n_{y, k}+n_{x} \alpha_{k}}{n_{\perp k}^{2}}, \quad h_{k}=\frac{i n_{y, k}-n_{x} \beta_{k}}{n_{\perp k}^{2}}, \quad k=1,2 .
\end{aligned}
$$

Since the tangential components of the electric field are continuous on the interface of a magnetoplasma and an isotropic medium, both the coefficients (22) and (23) and the coefficients (24) and (25) can be used when deriving the expressions for these field components on the surface $y=0$. In what follows, we use the coefficients (22) and (23). In view of Eqs. (22) (23), (26), and (27), as well as Eq. (17), rigorous integral representations corresponding to the current given by Eq. (6) can be obtained for the field components $E_{x}\left(n_{x}, y, z\right)$ and $E_{z}\left(n_{x}, y, z\right)$ at $y=0$ :

$$
E_{x}\left(n_{x}, 0, z\right)=\frac{i Z_{0} k_{0}}{2 \pi} \int_{-\infty}^{+\infty}\left[\int_{-d}^{d} \mathcal{I}\left(n_{x}, z^{\prime}\right) \exp \left(i k_{0} n_{z} z^{\prime}\right) \mathrm{d} z^{\prime}\right] \sum_{k=1}^{2} \frac{e_{k} \tilde{B}_{k}}{\Delta\left(n_{x}, n_{z}\right)} \exp \left(-i k_{0} n_{z} z\right) \mathrm{d} n_{z}
$$




$$
E_{z}\left(n_{x}, 0, z\right)=\frac{i Z_{0} k_{0}}{2 \pi \eta} \int_{-\infty}^{+\infty}\left[\int_{-d}^{d} \mathcal{I}\left(n_{x}, z^{\prime}\right) \exp \left(i k_{0} n_{z} z^{\prime}\right) \mathrm{d} z^{\prime}\right] \sum_{k=1}^{2} \frac{n_{k} \tilde{B}_{k}}{\Delta\left(n_{x}, n_{z}\right)} \exp \left(-i k_{0} n_{z} z\right) \mathrm{d} n_{z}
$$

where the coefficients $\tilde{B}_{k}(k=1,2)$ are described by the following formulas:

$$
\begin{gathered}
\tilde{B}_{1}=i h_{2} \frac{\eta}{\varepsilon_{\mathrm{a}}} \frac{n_{2} n_{y}}{n_{\perp}^{2}}-e_{2} \frac{\eta}{\varepsilon_{\mathrm{a}}} \frac{n_{x} n_{z}}{n_{\perp}^{2}}-n_{2} \frac{\varepsilon_{\mathrm{a}}-n_{x}^{2}}{\varepsilon_{\mathrm{a}} n_{\perp}^{2}}, \\
\tilde{B}_{2}=-i h_{1} \frac{\eta}{\varepsilon_{\mathrm{a}}} \frac{n_{1} n_{y}}{n_{\perp}^{2}}+e_{1} \frac{\eta}{\varepsilon_{\mathrm{a}}} \frac{n_{x} n_{z}}{n_{\perp}^{2}}+n_{1} \frac{\varepsilon_{\mathrm{a}}-n_{x}^{2}}{\varepsilon_{\mathrm{a}} n_{\perp}^{2}} .
\end{gathered}
$$

Changing the integration order in Eqs. (28) and (29), we arrive at the following expressions for the tangential field components $E_{x}(x, y, z)$ and $E_{z}(x, y, z)$ on the interface $y=0$ :

$$
\begin{aligned}
& E_{x}(x, 0, z)=\frac{k_{0}}{2 \pi} \int_{-\infty}^{+\infty} \mathrm{d} n_{x} \int_{-d}^{d} K_{x}\left(n_{x}, z-z^{\prime}\right) \mathcal{I}\left(n_{x}, z^{\prime}\right) \exp \left(-i k_{0} n_{x} x\right) \mathrm{d} z^{\prime} \\
& E_{z}(x, 0, z)=\frac{k_{0}}{2 \pi} \int_{-\infty}^{+\infty} \mathrm{d} n_{x} \int_{-d}^{d} K_{z}\left(n_{x}, z-z^{\prime}\right) \mathcal{I}\left(n_{x}, z^{\prime}\right) \exp \left(-i k_{0} n_{x} x\right) \mathrm{d} z^{\prime} .
\end{aligned}
$$

Here,

$$
\begin{gathered}
K_{x}\left(n_{x}, \zeta\right)=\frac{i Z_{0} k_{0}}{2 \pi} \int_{-\infty}^{+\infty} \sum_{k=1}^{2} \frac{e_{k} \tilde{B}_{k}}{\Delta\left(n_{x}, n_{z}\right)} \exp \left(-i k_{0} n_{z}|\zeta|\right) \mathrm{d} n_{z}, \\
K_{z}\left(n_{x}, \zeta\right)=\operatorname{sign}(\zeta) \frac{i Z_{0} k_{0}}{2 \pi \eta} \int_{-\infty}^{+\infty} \sum_{k=1}^{2} \frac{n_{k} \tilde{B}_{k}}{\Delta\left(n_{x}, n_{z}\right)} \exp \left(-i k_{0} n_{z}|\zeta|\right) \mathrm{d} n_{z}
\end{gathered}
$$

are the kernels of integral representations of the field components $E_{x}(x, 0, z)$ and $E_{z}(x, 0, z)$, respectively.

From boundary conditions (8) and (9) for the tangential components of the electric field on the antenna surface with allowance for Eqs. (4), (5), (31), and (32), integral equations can be obtained for the Fourier transform $\mathcal{I}\left(n_{x}, z\right)$ of the surface current density. Thus, from Eq. (8) we have

$$
\int_{-d}^{d} K_{x}\left(z-z^{\prime}\right) \mathcal{I}\left(n_{x}, z^{\prime}\right) \mathrm{d} z^{\prime}=-\mathcal{E}_{x}^{\operatorname{ext}}\left(n_{x}\right)
$$

Boundary condition (9) yields

$$
\int_{-d}^{d} K_{z}\left(z-z^{\prime}\right) \mathcal{I}\left(n_{x}, z^{\prime}\right) \mathrm{d} z^{\prime}=0
$$

In integral equations (35) and (36), it is assumed that $|z|<d$.

The behavior of solutions of the obtained integral equations is determined by the properties of their kernels. It is shown in what follows that in the case of a fairly small antenna width $2 d$, where the inequalities

$$
d \ll 2 \Delta, \quad\left(k_{0} d\right)^{2} \max \left\{\left|\varepsilon_{\mathrm{a}}\right|,|\varepsilon|,|g|,|\eta|\right\} \ll 1,
$$

are fulfilled, the properties of these kernels allow an approximate solution of Eqs. (35) and (36) to be obtained 
in analytical form. Note that the integrals in Eqs. (35) and (36), which are singular for $z^{\prime} \rightarrow z$, should be understood in the sense of the principal value.

\section{SOLVING INTEGRAL EQUATIONS FOR THE CURRENT}

We start the analysis of Eqs. (35) and (36) with studying the properties of their kernels (33) and (34). We represent the kernels of these equations in the form

$$
K_{x}\left(n_{x}, \zeta\right)=K_{x}^{(\mathrm{s})}\left(n_{x}, \zeta\right)+F_{x}\left(n_{x}, \zeta\right), \quad K_{z}\left(n_{x}, \zeta\right)=K_{z}^{(\mathrm{s})}\left(n_{x}, \zeta\right)+F_{z}\left(n_{x}, \zeta\right),
$$

where $K_{x}^{(\mathrm{s})}\left(n_{x}, \zeta\right)$ and $K_{z}^{(\mathrm{s})}\left(n_{x}, \zeta\right)$ are the singular parts of the kernels $K_{x}\left(n_{x}, \zeta\right)$ and $K_{z}\left(n_{x}, \zeta\right)$, respectively, whereas $F_{x}\left(n_{x}, \zeta\right)$ and $F_{z}\left(n_{x}, \zeta\right)$ are their regular parts. We take into account that in the considered case of resonant plasma (see Eq. (2)), the relations $\varepsilon>0$ and $\eta<0$ take place for the diagonal elements of the dielectric permittivity tensor given by Eq. (1). Under the additional condition $|\varepsilon \eta| \gg \varepsilon_{\mathrm{a}}^{2}$, which is usually fulfilled in the whistler frequency range (2) for the cases of practical interest, the singular parts of the kernels can be written in the form

$$
\begin{gathered}
K_{x}^{(\mathrm{s})}\left(n_{x}, \zeta\right)=\frac{i Z_{0} k_{0}}{2 \pi}\left[\left(-1+\frac{2 n_{x}^{2} \varepsilon_{\mathrm{a}}}{\varepsilon_{\mathrm{a}}^{2}+|\varepsilon \eta|}\right) \int_{\left|n_{x}\right|}^{+\infty} \frac{\sqrt{n_{z}^{2}+n_{x}^{2}}}{n_{z}^{2}} \cos \left(k_{0} n_{z}|\zeta|\right) \mathrm{d} n_{z}\right. \\
\left.+\frac{2 i n_{x}^{2}}{\sqrt{|\varepsilon \eta|}} \int_{\tilde{n}_{z}}^{+\infty} \frac{\cos \left(k_{0} n_{z}|\zeta|\right)}{\sqrt{n_{z}^{2}-|\varepsilon / \eta| n_{x}^{2}}} \mathrm{~d} n_{z}\right] \\
K_{z}^{(\mathrm{s})}\left(n_{x}, \zeta\right)=\frac{i Z_{0} k_{0}}{\pi \sqrt{|\varepsilon \eta|}} n_{x} \operatorname{sign}(\zeta) \int_{\tilde{n}_{z}}^{+\infty} \frac{n_{z} \sin \left(k_{0} n_{z}|\zeta|\right)}{\sqrt{n_{z}^{2}-|\varepsilon / \eta| n_{x}^{2}}} \mathrm{~d} n_{z},
\end{gathered}
$$

where $\tilde{n}_{z}=\sqrt{|\varepsilon / \eta|}\left|n_{x}\right|$. The regular parts $F_{x}\left(n_{x}, \zeta\right)$ and $F_{z}\left(n_{x}, \zeta\right)$ of the kernels are determined by integrals over $n_{z}$, whose integrands are given by the differences of the corresponding quantities in rigorous formulas for the kernels $K_{x}\left(n_{x}, \zeta\right)$ and $K_{z}\left(n_{x}, \zeta\right)$ and in Eqs. (39) and (40).

It can easily be shown that quantities (39) and (40) tend to infinity at $\zeta=0$, while the quantities $F_{x}\left(n_{x}, \zeta\right)$ and $F_{z}\left(n_{x}, \zeta\right)$ do not have singularities at this point. Thus, indeed we have obtained representations of the kernels $K_{x}\left(n_{x}, \zeta\right)$ and $K_{z}\left(n_{x}, \zeta\right)$ in the form of sums of the singular and nonsingular terms. In the latter, one can then put $\zeta=0$ if conditions (37) are fulfilled.

The integrals in Eqs. (39) and (40) can be calculated analytically [13], and under conditions (37) they are given by the expressions

$$
\begin{gathered}
K_{x}^{(\mathrm{s})}\left(n_{x}, \zeta\right)=-\frac{i Z_{0} k_{0}}{2 \pi}\left\{\left[-1+\frac{2 n_{x}^{2} \varepsilon_{\mathrm{a}}}{\varepsilon_{\mathrm{a}}^{2}+|\varepsilon \eta|}+\frac{2 i n_{x}^{2}}{\sqrt{|\varepsilon \eta|}}\right]\left(\ln \frac{k_{0}|\zeta|}{2}+\ln \left|n_{x}\right|+C\right)+\frac{2 i n_{x}^{2}}{\sqrt{|\varepsilon \eta|}} \ln \sqrt{|\varepsilon| /|\eta|}\right\}, \\
K_{z}^{(\mathrm{s})}\left(n_{x}, \zeta\right)=\frac{i Z_{0}}{\pi \sqrt{|\varepsilon \eta|}} \frac{n_{x}}{\zeta}
\end{gathered}
$$

where $C=0.5772 \ldots$ is Euler's constant.

After the algebra given above, integral equation (35) for $|z|<d$ can be written as follows:

$$
\int_{-d}^{d} \mathcal{I}\left(n_{x}, z^{\prime}\right) \ln \frac{k_{0}\left|z-z^{\prime}\right|}{2} \mathrm{~d} z^{\prime}=\frac{2 i \pi}{Z_{0} k_{0}} \chi \mathcal{E}_{x}^{\operatorname{ext}}\left(n_{x}\right)-S\left(n_{x}\right) \int_{-d}^{d} \mathcal{I}\left(n_{x}, z^{\prime}\right) \mathrm{d} z^{\prime}
$$


where

$$
\begin{gathered}
\chi=\frac{i \xi}{n_{x}^{2}+i \xi}, \quad \xi=\frac{\sqrt{|\varepsilon \eta|}}{2} /\left(1-\frac{i \varepsilon_{\mathrm{a}} \sqrt{|\varepsilon \eta|}}{\varepsilon_{\mathrm{a}}^{2}+|\varepsilon \eta|}\right), \\
S\left(n_{x}\right)=C+\ln \left|n_{x}\right|-\frac{2 i n_{x}^{2}}{\sqrt{|\varepsilon \eta|}} \chi \ln \sqrt{|\varepsilon| /|\eta|}-\frac{2 i \pi}{Z_{0} k_{0}} \chi F_{x}\left(n_{x}, 0\right) .
\end{gathered}
$$

Recall that the expression for $\xi$ was obtained under the condition $|\varepsilon \eta| \gg \varepsilon_{\mathrm{a}}^{2}$. Equation (36), in turn, when inequalities (37) are fulfilled, transforms to the form

$$
\int_{-d}^{d} n_{x} \frac{\mathcal{I}\left(n_{x}, z^{\prime}\right)}{z-z^{\prime}} \mathrm{d} z^{\prime}=0
$$

When deriving Eq. (46), it was taken into account that $F_{z}\left(n_{x}, 0\right)=0$, so that the kernel of Eq. (36) is determined by its singular part (42). It can be shown that the solutions of Eqs. (43) and (46) are the main terms of asymptotics of the solutions of the initial integral equations (35) and (36) when inequalities (37) are fulfilled. Then we restrict ourselves to analyzing only Eqs. (43) and (46).

The solution of Eq. (43) with a logarithmic kernel, which also satisfies Eq. (46) with Cauchy's kernel [14], can be obtained analytically and has the form

$$
\mathcal{I}\left(n_{x}, z\right)=-\frac{2 i}{Z_{0} k_{0} \sqrt{d^{2}-z^{2}}} \frac{\chi V_{0}}{\ln \left[4 /\left(k_{0} d\right)\right]-S\left(n_{x}\right)} \frac{\sin \left(k_{0} n_{x} \Delta\right)}{k_{0} n_{x} \Delta} .
$$

Substituting Eq. (47) into Eq. (7), we arrive at the following formula for the surface current density $I(x, z)$ in a strip antenna:

$$
I(x, z)=-\frac{i V_{0}}{Z_{0} \pi \sqrt{d^{2}-z^{2}}} \int_{-\infty}^{+\infty} \frac{\sin \left(k_{0} n_{x} \Delta\right)}{k_{0} n_{x} \Delta} \frac{\chi \exp \left(-i k_{0} n_{x} x\right)}{\ln \left[4 /\left(k_{0} d\right)\right]-S\left(n_{x}\right)} \mathrm{d} n_{x} .
$$

The total current in the cross section $x=$ const is determined by the relation

$$
I(x)=\int_{-d}^{d} I(x, z) \mathrm{d} z .
$$

Note that the singularity of the function $I(x, z)$ for $|z| \rightarrow d$ (see Eq. (48)), which corresponds to the Meixner condition at the edge [15], is integrable, so that the total current $I(x)$ of the antenna in the cross section $x=$ const is finite.

\section{RESULTS OF CALCULATIONS OF THE CURRENT DISTRIBUTION AND IMPEDANCE OF THE ANTENNA}

Even after all the assumptions, integral representation (48) admits only a numerical study. However, if the condition $\ln \left[4 /\left(k_{0} d\right)\right] \gg\left|S\left(n_{x}\right)\right|$ is fulfilled for the values $\left|n_{x}\right|<\left(k_{0} \Delta\right)^{-1}$, then the integral in Eq. (48) can be evaluated analytically, and the distribution of current along the antenna takes the form

$$
I(x)=\frac{V_{0} \pi h}{Z_{0} k_{0} \ln \left[4 /\left(k_{0} d\right)\right]} \exp (-i h|x|),
$$




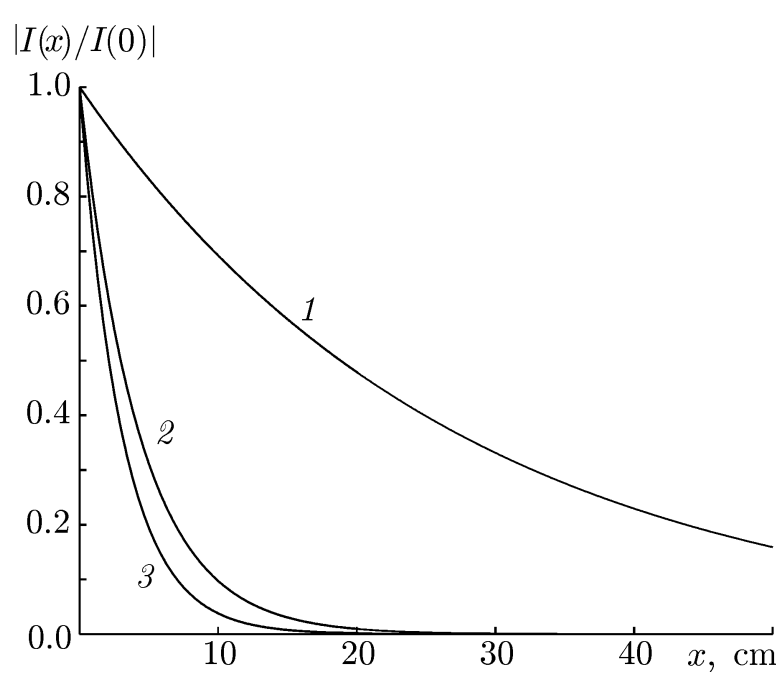

Fig. 2. Dependences of the normalized current amplitude on the $x$ coordinate for $N=10^{11} \mathrm{~cm}^{-3}$ (curve 1), $N=5 \cdot 10^{12} \mathrm{~cm}^{-3}$ (curve 2), and $N=10^{13} \mathrm{~cm}^{-3}$ (curve 3) for $d=0.01 \mathrm{~cm}, \varepsilon_{\mathrm{a}}=1$, $B_{0}=800 \mathrm{G}$, and $\omega=1.7 \cdot 10^{8} \mathrm{~s}^{-1}$.

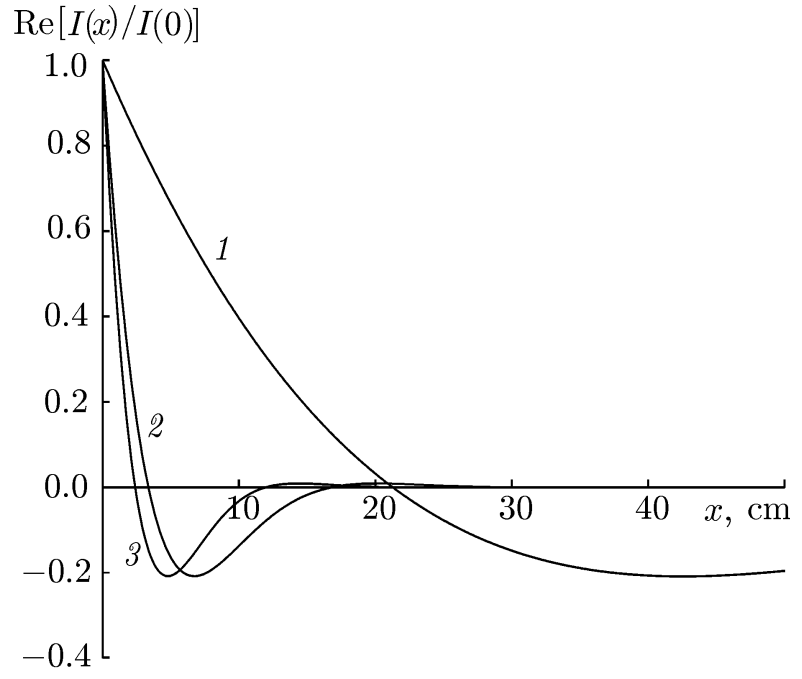

Fig. 3. Dependences of the normalized quantity $\operatorname{Re} I(x)$ on the $x$ coordinate for $N=10^{11} \mathrm{~cm}^{-3}$ (curve 1), $N=5 \cdot 10^{12} \mathrm{~cm}^{-3}$ (curve 2), and $N=$ $10^{13} \mathrm{~cm}^{-3}$ (curve 3) for the same values of the parameters $d, \varepsilon_{\mathrm{a}}, B_{0}$, and $\omega$ as in Fig. 2 .

where $|x|>\Delta$ and

$$
h=k_{0} \frac{1-i}{\sqrt{2}} \sqrt{\xi} .
$$

The obtained approximate formula (50) obviously corresponds to the transition to the transmission line theory and gives a complex propagation constant $h$ of the antenna current. Thus, the conditions under which this formula was obtained determines in fact the limits of applicability of the transmission line theory for a linear antenna located on the interface of an isotropic medium and a resonant magnetoplasma. It should be mentioned that in the case considered here, i. e., for $|\varepsilon \eta|^{1 / 2} \gg \varepsilon_{\mathrm{a}}$, the current propagation constant $h$ is given approximately by the formula $h \approx k_{0}(1-i)|\varepsilon \eta|^{1 / 4} / 2$ and is a factor of $\sqrt{2}$ smaller than the corresponding quantity for the antenna located in a homogeneous resonant magnetoplasma [8].

Using the current distribution $I(x)$, we write the input impedance $Z=R+i X$ of the antenna. It can be obtained in a standard way using the formula $Z=V_{0} / I(\Delta)$ and, within the framework of approximation (50) under the condition $|h| \Delta \ll 1$, is determined by the expression

$$
Z=\frac{Z_{0}}{\pi} \frac{k_{0}}{h} \ln \left(\frac{4}{k_{0} d}\right)
$$

We now proceed to the results of numerical calculations, which illustrate the behavior of the current distribution and input impedance of the strip antenna considered. The calculations were performed for the following values of the parameters corresponding to the laboratory plasma conditions: $1.78 \cdot 10^{10} \mathrm{~s}^{-1} \leq \omega_{\mathrm{p}} \leq$ $1.78 \cdot 10^{11} \mathrm{~s}^{-1}$ (the plasma density $N$ lies in the interval $10^{11} \mathrm{~cm}^{-3} \leq N \leq 10^{13} \mathrm{~cm}^{-3}$ ), $\omega_{H}=1.41 \cdot 10^{10} \mathrm{~s}^{-1}$ (external magnetic field $B_{0}=800 \mathrm{G}$ ), and signal frequency $\omega=1.7 \cdot 10^{8} \mathrm{~s}^{-1}$ lies in the whistler frequency range (2). In the calculations, it was assumed that the strip conductor half-width $d=0.01 \mathrm{~cm}, \Delta=0.05 \mathrm{~cm}$, the center of the region to which the external voltage is applied has the coordinate $x_{0}=0$, and the relative dielectric permittivity of the isotropic medium for $y>0$ amounts to $\varepsilon_{\mathrm{a}}=1$ (free space).

The dependences of the normalized (to the current at the point $x=0$ ) quantities $|I(x)|$ and $\operatorname{Re} I(x)$ are shown in Figs. 2 and 3, respectively, for three values of the plasma density, namely, $N=10^{11}, 5 \cdot 10^{12}$, and $10^{13} \mathrm{~cm}^{-3}$. For the chosen parameters, the results of calculations by Eq. (48) and approximate formula (50) 
in the limit $\Delta \rightarrow 0$ coincide with graphical accuracy. It is seen in Fig. 2 that the current amplitude decreases rapidly along the antenna wire with increasing distance from the point of application of the external voltage, and this decrease is the faster the higher is the plasma density.

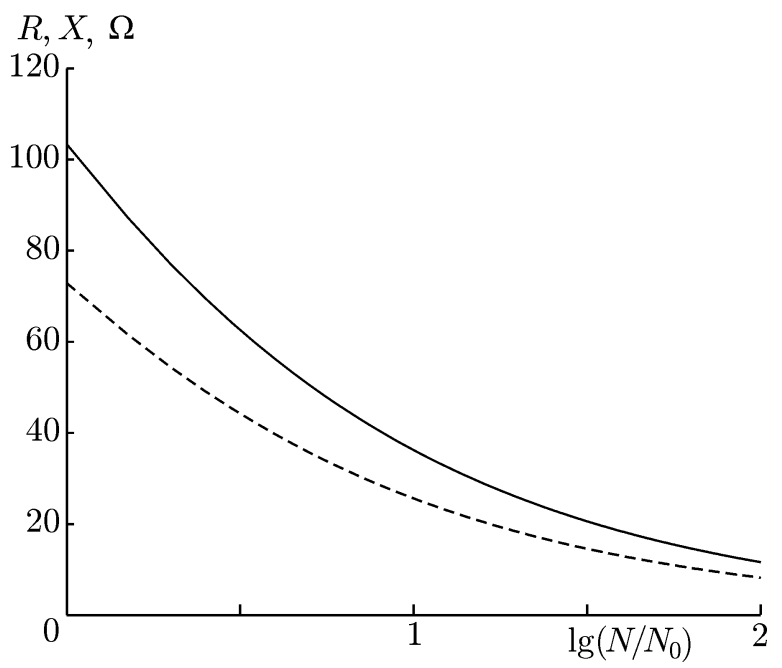

Fig. 4. Dependences of the radiation resistance $R$ and reactance $X$ of the antenna on the normalized plasma density in cases where the antenna is located on a plane interface of a magnetoplasma and free space (solid line) and in a homogeneous magnetoplasma (dashed line) for $N_{0}=10^{11} \mathrm{~cm}^{-3}$ and the same values of other parameters as in Fig. 2.

current can be written as follows:
Figure 4 shows the dependences of the radiation resistance $R=\operatorname{Re} Z$ and reactance $X=\operatorname{Im} Z$ of the antenna on the normalized plasma density $N / N_{0}$ (here, $N_{0}=10^{11} \mathrm{~cm}^{-3}$ ), which were obtained using Eq. (52) for the above-mentioned values of other parameters. Note that within the framework of approximation (52), the real and imaginary parts of the input impedance of the antenna turn out to be identical, i. e., $R=X$. In addition, the dashed line in Fig. 4 shows the real and imaginary parts of the input impedance of the same antenna located in a homogeneous unbounded plasma with the same values of the parameters (see [8]). It is seen in Fig. 4 that the dependences of $R$ and $X$ in the presence of an interface in the case considered are similar to the behavior of the corresponding quantities for the antenna in a homogeneous unbounded plasma, but the the values of $R$ and $X$ are greater than in the case of a homogeneous plasma medium.

It should be mentioned that the results obtained in this paper can easily be applied to a finite-length antenna, which can be described as a transmission line of length $2 L$. Within the framework of this approximation, the antenna

$$
I(x)=I_{0}\left[\exp (-i h|x|)+R_{1} \exp (-i h x)+R_{2} \exp (i h x)\right]
$$

where $|x|<L, I_{0}$ is a certain constant, and $h$ is the current propagation constant in an infinitely long antenna (see Eq. (51)). The coefficients $R_{1}$ and $R_{2}$ can be found from the condition that the current is equal to zero at the ends $x= \pm L$ of the antenna. As a result, we obtain the following formula for the current distribution along the antenna:

$$
I(x)=\frac{I(0)}{\sin (h L)} \sin [h(L-|x|)], \quad|x|<L,
$$

where $I(0)$ is the current at the antenna input.

In the case of an electrically short antenna, where the condition $|h| L \ll 1$ is fulfilled, from Eq. (54) it is easy to obtain a "triangular" distribution of current along the antenna wire $(|x|<L)$ :

$$
I(x)=I(0)(1-|x| / L) .
$$

In the opposite case of a long antenna $(|h| L \gg 1)$, the current distribution given by Eq. (54) transforms (with allowance for the inequality $\operatorname{Im} h<0$ ) to an exponential distribution of Eq. (50).

The input impedance $Z$ of a finite-length antenna can be determined in a standard way [1] on the basis of the found current distribution using the induced EMF method:

$$
Z=-\int \hat{\mathbf{J}}^{*}(\mathbf{r}) \hat{\mathbf{E}}(\mathbf{r}) \mathrm{d} \mathbf{r}
$$


where $\hat{\mathbf{J}}=\mathbf{J} / I(0)$ is the density of the antenna current normalized to $I(0), \hat{\mathbf{E}}$ is the field excited by this normalized current, and the asterisk denotes complex conjugation. Recall that the value of the integral in Eq. (56) is determined by the form of the current distribution and does not depend on $I(0)$. It is noteworthy that the use of Eq. (54) with a complex propagation constant given by Eq. (51) for calculation of the input impedance of the antenna does not lead to an infinite value of $Z$ that takes place within the framework of the transmission line theory for $h L=\pi n(n=1,2, \ldots)$ in cases where the quantity $h$ is real [5]. The nonzero value of the imaginary part of $h$ is due to the fact that the loss for radiation of small-scale quasielectrostatic waves $[1,4]$ to the half-space $y<0$ filled with a resonant magnetoplasma was taken into account when Eq. (51) was derived.

After the calculation of $Z$, the current at the input of a finite-length antenna can be found using the formula $I(0)=V_{0} / Z$. Leaving aside the calculation of the input impedance $Z$ by Eq. (56), we only note that increasing the antenna wire length up to values significantly exceeding the characteristic scale $|h|^{-1}$ of the current decrease along the antenna is inexpedient since this is not accompanied by an increase in the real part of the input impedance of the antenna and, therefore, its radiated power.

\section{CONCLUSIONS}

We have considered the problem of the current distribution of a perfectly conducting strip antenna that is perpendicular to the external magnetic field and located on a plane interface of an isotropic medium and a resonant magnetosplasma, which is described by a dielectric permittivity tensor of general form. In the case of an infinitely long antenna, the problem was reduced to a system of singular integral equations for the current. As a result of the solution of these equations, we obtained expressions for the current distribution and input impedance of the antenna. It is shown that under certain conditions, which establish the limits of applicability of the approximate method based on the transmission line theory, we succeeded in obtaining relatively simple expressions for elecrodynamic characteristics of the antenna considered. In such an approximation, the current distribution, as well as the input impedance of the antenna located on the interface of a resonant magnetoplasma and an isotropic medium coincide with the corresponding characteristics of a certain equivalent transmission line with a complex current propagation constant. Moreover, the results obtained for an infinitely long antenna can easily be generalized to the case of a finite-length antenna within the framework of the transmission line theory. The approach used in this paper can be employed for determining the electrodynamic characteristics of the antenna located not only on an interface of an isotropic medium and a magnetosplasma, but also on an interface of arbitrary anisotropic media.

The study of the current distribution of an infinitely long antenna was supported by the Russian Science Foundation (project No. 14-12-00510). Other results were obtained under support of the Russian Foundation for Basic Research (project No. 14-01-31280), the grant of the President of the Russian Federation for young $\mathrm{PhD}$ researchers (project No. MK-4688.2014.2), and the grant of the Russian Government (contract No. 11.G34.31.0048).

\section{REFERENCES}

1. E. A. Mareev and Yu. V. Chugunov, Antennas in Plasmas [in Russian], Inst. Appl. Phys., Nizhny Novgorod (1991).

2. V.V.Akindinov, S. M. Eremin, and I. V.Lishin, Radiotekh. Élektron., 30, No. 5, 833 (1985).

3. R. L. Stenzel, Radio Sci., 11, No. 12, 1045 (1976).

4. I. G. Kondrat'ev, A. V. Kudrin, and T. M.Zaboronkova, Electrodynamics of Density Ducts in Magnetized Plasmas, Gordon and Breach, Amsterdam (1999).

5. S. Adachi, T. Ishizone, and Y. Mushiake, Radio Sci., 12, No. 1, 23 (1977).

6. S. Ohnuki, K. Sawaya, and S. Adachi, IEEE Trans. Antennas Propagat., 34, No. 8, 1024 (1986). 
7. A. V. Kudrin, E. Yu.Petrov, and T. M.Zaboronkova, J. Electromagn. Waves Appl., 15, No. 3, 345 (2001).

8. T. M.Zaboronkova, A. V. Kudrin, and E. Yu.Petrov, J. Commuin. Technol. Electron., 57, No. 3, 296 (2012).

9. A. V. Kostrov, A. V. Kudrin, L. E. Kurina, et al., Phys. Scr., 62, Pt. 1, 51 (2000).

10. A. V. Kudrin, A. S. Zaitseva, T. M. Zaboronkova, et al., Prog. Electromagn. Res. B, 51, 221 (2013).

11. A. V. Kudrin, A. S. Zaitseva, T. M. Zaboronkova, and S. S. Zilitinkevich, Prog. Electromagn. Res. B, 55, 241 (2013).

12. V.L.Ginzburg, The Propagation of Electromagnetic Waves in Plasmas, Pergamon Press, Oxford (1970).

13. A.P. Prudnikov, Yu. A. Brychkov, and O.I. Marichev, Integrals and Series, Special Functions [in Russian], Nauka, Moscow (1983).

14. V.V. Vorovich, V. M. Aleksandrov, and V. A. Babeshko, Nonclassical Mixed Problems in the Theory of Elasticity [in Russian], Nauka, Moscow (1974), p. 215.

15. J. Meixner, IEEE Trans. Antennas Propagat., 20, No. 4, 442 (1972). 